\title{
Homo-dimerization of RyR1 C-terminus via charged residues in random coils or in an $\alpha$-helix
}

\author{
Eun Hui Lee ${ }^{1,3}$ and Paul D. Allen ${ }^{2}$ \\ ${ }^{1}$ Department of Physiology, College of Medicine \\ The Catholic University of Korea \\ Seoul 137-701, Korea \\ 2Department of Anesthesiology, Perioperative and Pain Medicine \\ Brigham and Women's Hospital, Harvard Medical School \\ Boston, MA 02115, USA \\ ${ }^{3}$ Corresponding author: Tel, 82-2-590-4990; \\ Fax, 82-2-532-9575; E-mail, EHUI@ catholic.ac.kr
}

Accepted 23 July 2007

Abbreviations: RyR, ryanodine receptor; wt, wild type

\begin{abstract}
To investigate the mechanism by which the $\mathrm{C}$-terminus $(4,938-5,037)$ of the ryanodine receptor 1 (RyR1) homo-tetramerizes, forming a functional $\mathrm{Ca}^{2+}$-release channel, the structural requirements for the tetramerization were studied using site-directed mutagenesis. Alanine-substitutions at five charged residues, E4976, H5003, D5026, E5033 and D5034, significantly decreased the formation of homo-dimers (reduced by $>$ $50 \%$ ). Interaction between the $\mathrm{C}$-terminus and cytoplasmic loop I $(4,821-4,835)$ required two positively charged residues, $\mathrm{H} 4832$ and K4835. Based on the predicted protein secondary structures, all seven charged residues are located in random coils. Paired alanine-substitutions at six negatively charged residues (E4942A/D4953A, D4945A/E4952A and E4948A/ E4955A) of the $\alpha$-helix $(4,940-4,956)$ in the $C$-terminus increased homo-dimerization. Therefore, the homo-tetramerization of RyR1 may be mediated by intraand/or inter-monomer electrostatic interactions among the C-terminal charged residues in random coils or in an $\alpha$-helix.
\end{abstract}

Keywords: mutagenesis, site-directed; ryanodine receptor calcium release channel; structure-activity relationship

\section{Introduction}

The ryanodine receptors $\left(\mathrm{Ca}^{2+}\right.$-release channels,
RyRs) in the sarcoplamsic reticulum (SR) are homo-tetrameric proteins composed of four individual monomers (Sandow, 1965). Three RyR isoforms arising from three separate genes have been identified: RyR1 (skeletal isoform), RyR2 (cardiac isoform) and RyR3 (brain isoform). RyR1 and RyR2 are essential proteins for excitationcontraction coupling in striated muscles, such as skeletal and cardiac muscle, functioning as $\mathrm{Ca}^{2+}$ release channels in the SR. RyR3 is a ubiquitous protein with much lower expression levels relative to those of RyR1 and RyR2; the functional role of RyR3 in the cells in which it is expressed remains undefined (Ogawa et al., 2000).

In rabbits, the three RyR isoforms show $67 \%$ (RyR1-RyR2), 67\% (RyR1-RyR3) and 70\% (RyR2RyR3) amino acid sequence identity (Hakamata et al., 1992), and there is a similar degree of identity among other mammalian isoforms (Sandow, 1965; Zorzato et al., 1990; Williams et al., 2001; Samso et al., 2005). RyR is assumed to be composed of a large cytoplasmic foot assembly (N-terminal 3/4 $4 / 5$ ), a smaller transmembrane (TM) assembly (Cterminal 1/4 1/5) and a short cytoplasmic C-terminus domain (100 amino acids). The amino acid sequences of the $\mathrm{C}$-terminus of the three rabbit RyR isoforms show extremely high homology $(\geq 94 \%)$.

The C-terminus of RyR2 appears to be important for the homo-tetramerization of RyR2 (Stewart et al., 2003), and the deletion of the last 15 amino acids from a full-length RyR1 cDNA produces a non-functional protein (Gao et al., 1997). In support of the hypothesis that the C-terminus is important in the homo-tetramerization of RyR1 and RyR2, the critical domains for homo-tetramerization of $\mathrm{IP}_{3}$ receptors $\left(\mathrm{IP}_{3} \mathrm{Rs}\right)$ - sister ER $\mathrm{Ca}^{2+}$-release channels to RyRs - are located both in the C-terminus and in the fifth and sixth TM domains (Galvan et al., 1999). In addition, self-interaction is observed in the cytoplasmic region preceding the first TM domain by yeast two-hybrid studies (Magnino et al., 2001). In the case of shaker- and shaw-type $\mathrm{K}^{+}$ channels, homo-tetramerization is mediated by $\mathrm{Zn}^{2+}$ ions coordinated with cysteine and histidine residues of the T1 domain localized in the hydrophilic N-terminal region (Bixby et al., 1999; Jahng et al., 2002). Therefore, based on observation of these very different mechanisms, it is possible that each homo-tetrameric channel family evolved a 
unique way to assemble functional oligomeric channels and these differences have been preserved.

In the current study, the mechanisms governing homo-tetramerization of RyR1 by its C-terminus were examined using site-directed mutagenesis coupled with biochemical assays. The charged residues located in random coils or in the $\alpha$-helix of C-terminal RyR1 were critical to homo-dimerization of the C-terminus, suggesting that RyR1 homo -tetramerization via its C-terminus was possibly mediated by intra- and/or inter-electrostatic interactions among the charged residues located in the C-terminal random coils and the $\alpha$-helix.

\section{Materials and Methods}

\section{Materials}

Isopropyl- $\alpha$-D-thiogalacto-pyranoside (IPTG) and $\mathrm{ZnSO}_{4}$ were obtained from Sigma-Aldrich (St. Louis, MO). Monoclonal anti-RyR1 antibody (34C) was provided by Drs. J. Airey and J. Sutko (Developmental Studies Hybridoma Bank, lowa City, IA). Anti-His and anti-GST antibody were obtained from Santa Cruz Biotechnology (Santa Cruz, CA). Glutathione-Sepharose 4B (GST) and protein GSepharose 4 Fast Flow affinity bead were obtained from Amersham Biosciences (Piscataway, NJ).

\section{Mutation and expression of the RyR1 C-terminal fragments}

Using rabbit RyR1 cDNA, C-terminal portions depicted in Figure 4 were sub-cloned into a GST vector ( $p G E X$, Amersham Biosciences, Piscataway, NJ) and/or a His-tag vector, (pRSET A, Invitrogen Corporation, Carlsbad, CA) between BamHI and Xhol sites. Mutations of GST- or His-tagged constructs were carried out using site-directed mutagenesis system (QuickChange site-directed mutagenesis kit, Stratagene, La Jolla, CA) with desired mis-matched oligonucleotides sets. Production of GST- or His-tagged proteins were induced in log phase $E$. coli by the addition of $0.1 \mathrm{mM}$ IPTG for $8 \mathrm{~h}$ at $37^{\circ} \mathrm{C}$, and each bacterial cell lysate was prepared by sonication in $\mathrm{PBS}$ (2.71 $\mathrm{mM} \mathrm{Na}_{2} \mathrm{HPO}_{4}, 1.54 \mathrm{mM} \mathrm{KH}_{2} \mathrm{PO}_{4}, 155 \mathrm{mM}$ $\mathrm{NaCl}$ and $\mathrm{pH}$ 7.2) with protease inhibitors (1 $\mu \mathrm{M}$ pepstatin, $1 \mu \mathrm{M}$ leupeptin, $100 \mu \mathrm{M}$ PMSF and 1 $\mu \mathrm{M}$ trypsin inhibitor). After centrifugation of the bacterial cell lysate at $7,700 \mathrm{~g}$ for $1 \mathrm{~min}$, protein concentration of the supernatant was determined by Bradford method and used for further experiments.

\section{Co-immunoprecipitation and GST pull-down assays}

For the co-immunoprecipitation assay, heavy SR vesicles were prepared from rabbit fast-twitch muscle (back and leg), using a procedure described elsewhere (Lee et al., 2004), and were solubilized in a buffer solution ( $1 \%$ Triton X-100, 10 $\mathrm{mM}$ Tris, $150 \mathrm{mM} \mathrm{NaCl}, 5 \mathrm{mM}$ EDTA, $1 \mathrm{mM}$ $\mathrm{Na}_{3} \mathrm{VO}_{4}, 10 \%$ glycerol, protease inhibitors, $\mathrm{pH}$ 7.4). The solubilized SR $(500 \mu \mathrm{g}$ total protein) were incubated with bacterial lysate containing wild type His-tag C-terminus (wtHis-A) or GST-tag cytoplasmic loop I (wtGST-C) for $4 \mathrm{~h}$ at $4^{\circ} \mathrm{C}$, and incubated with $30 \mu \mathrm{l}$ of anti-RyR 1 antibody overnight at $4^{\circ} \mathrm{C}$, followed by further incubation with protein GSepharose 4 Fast Flow affinity beads (Amersham Biosciences) for $4 \mathrm{~h}$ at $4^{\circ} \mathrm{C}$. For the GST pull-down assay, bacterial lysate, expressing one of the GST-tag proteins, was incubated with bacterial lysate, expressing one of the His-tag proteins, for 4 $\mathrm{h}$ at $4^{\circ} \mathrm{C}$, and incubated with GST beads (Amersham Biosciences) for $4 \mathrm{~h}$ at $4^{\circ} \mathrm{C}$. For both assays, the bead-complexes were then washed three times with PBS to remove non-specifically bound proteins, then loaded on 8 or $15 \%$ polyacrylamide gels and analyzed by immunoblot analysis.

\section{Native and modified-native PAGE}

A Laemmli gel system (Laemmli, 1970) without SDS was used for native PAGE. For modifiednative PAGE, the amount of reducing reagent was decreased by $60 \%$ or $80 \%$ compared with a standard SDS-PAGE $(0.04 \%$ SDS in polyacrylamide gel and tris-glycine running buffer, $0.2 \%$ SDS and $2 \% 2$-mercapotoethanol in sample buffer).

\section{Prediction of secondary protein structure}

Secondary structures were predicted using a computer software program, PSIpred (v2.5), from Brunel University (McGuffin et al., 2000). The confidence of each residue for the predicted secondary structure was scored using confidencevalues ranging from 0 (low) to 9 (high).

\section{Statistical analysis}

Results are given as means \pm S.E. of at least three independent experiments. Significant differences were analyzed using the paired $t$-test (GraphPad InStat, v2.04). Differences were considered to be significant at $P<0.05$. 


\section{Results and Discussion}

To examine the mechanism by which four RyR1 monomers tetramerize via their $\mathrm{C}$-terminus to form a single functional $\mathrm{Ca}^{2+}$-release channel, the C-terminus of RyR1 (100 amino acids at positions 4,938-5,037) (Figure 1A) was cloned as a $\mathrm{N}$ terminal His-tag protein, expressed in bacteria, and then subjected to native PAGE followed by immunoblot analysis with anti-His antibody (Figure 1B). Regardless of sample boiling duration ( 0 to 15 min), these gels showed that the wild type His-tag C-terminus (wtHis-C-terminus) formed homo-tetrameric and multiple homo-oligomeric complexes with only trace amounts of monomer or dimer. The amount of reducing reagent was significantly lowered compared to standard SDS-PAGE (60$80 \%$ reduction in the modified-native PAGE as described in Materials and Methods) to allow detection of the transition from monomer to dimer, trimer or tetramer. For a control experiment, GST (glutathione S-transferases), a well-known dimeric protein in cytoplasm (Rushmore and Pickett, 1993), was subjected to the modified-native PAGE and immunoblot analysis with anti-GST antibody, and we confirmed the transition of GST from monomer to dimer, tetramer or multimer in the modified-native PAGE (Supplemental data 1). Under these conditions, wtHis-C-terminus existed mainly in the monomer and dimer forms rather than as multiple oligomers (Figure 1C). All further experiments were conducted using the modifiednative PAGE.

\section{The role of charged residues in the homo-dimerization of the $\mathrm{C}$-terminus}

Homo-tetramerization of $\mathrm{K}^{+}$channels (Bixby et al., 1999; Jahng et al., 2002) occurs via intercoordination by $\mathrm{Zn}^{2+}$ ions at cysteine and histidine residues. To determine if homo-tetramerization of RyR1 proceeds via a similar mechanism, the

\section{A}

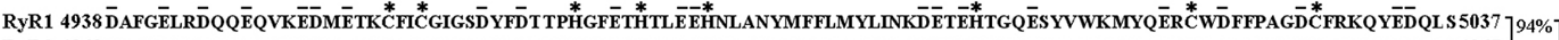
RYR2 4868 DAFGELRDQQEQVKEDMETKCFICGIGNDYFDTVPHGFETHTLQEHNLANYLFFLMYLINKDETEHTGQES YVWKMYQERCWEFFPAGDCFRKQYEDQLN4967 RYR3 4771 DAFGELRDQQEQVREDMETKC FICGIGNDYFDTTPHGFETHTLQEHNLANYLFFLMYLINKDETEHTGQES YVWKMYQERCWDFFPAGDCFRKQYEDQLG4870 $\left.]_{96 \%}\right]$

B

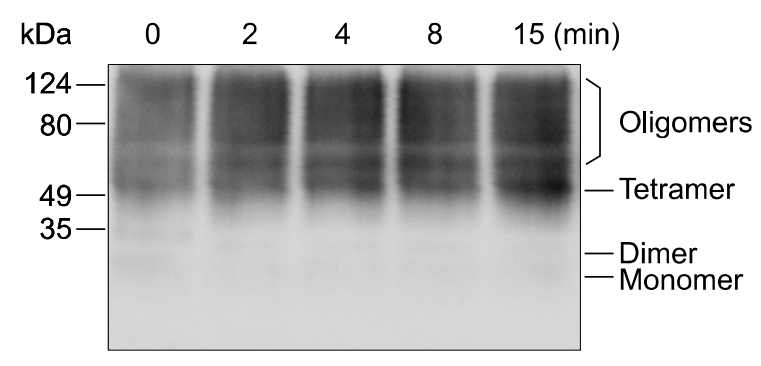

D

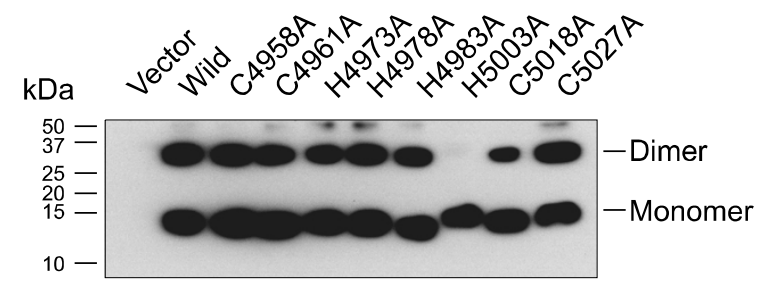

C

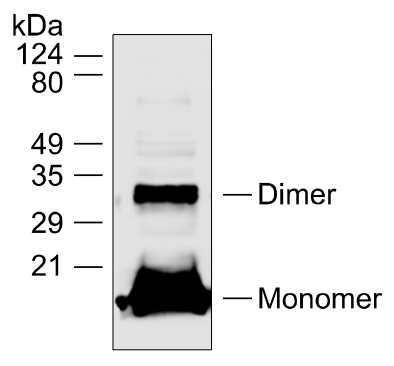

E

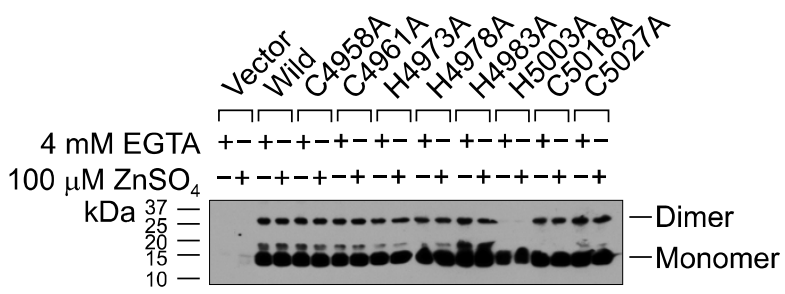

Figure 1. The effect of single mutations at cysteine or histidine residues on the homo-dimerization of the C-terminus. (A) Sequence comparison of the C-termini of rabbit RyR1, RyR2 and RyR3 ( $\geq 94 \%$ identity). The absolutely conserved 4 cysteines and 4 histidines $(\mathrm{C}$ and $\mathrm{H}$ ) are indicated by asterisks. The 21 conserved negatively charged aspartic and glutamic acids ( $D$ and $E$ ) are indicated by short bars. (B) Oligomerization of wtHis-C-terminus was examined using native PAGE and immunoblot analysis with anti-His antibody. Each sample was boiled with sample buffer for 0 to 15 min. (C) wtHis-C-terminus was subjected to modified-native PAGE, as described in Materials and methods, and to immunoblot analysis. (D) Modified-native PAGE and immunoblot analysis of individual mutants at cysteines or histidines of the $\mathrm{C}$-terminus. $(\mathrm{E})$ The effects of $\mathrm{Zn}^{2+}$ ions on the homo-dimerization of wtHis-C-terminus and each of $8 \mathrm{C}$-terminal mutants ( $4 \mathrm{mM} \mathrm{EGTA}$ or $100 \mu \mathrm{M} \mathrm{ZnSO} 4$ ). Wild is the wtHis-C-terminus, as a positive control. Vector is His-tag only, as a negative control. 
C-terminal cysteine and histidine (indicated by asterisks in Figure 1A) were substituted with alanines. The mutants were expressed in bacteria and then subjected to modified-native PAGE, followed by immunoblot analysis (Figure 1D). To eliminate the formation of His-tag oligomeric complexes, expression of His-tag only was used as a negative control (Vector in Figure $1 D$ and $E$ ). Among the eight cysteine or histidine mutants, H5003A nearly abolished the ability of the Cterminus to homo-dimerize. The other seven mutants showed a degree of homo-dimerization similar to that of wtHis-C-terminus (Wild in Figure $1 D$ and $E)$. In addition, the presence or absence of $\mathrm{Zn}^{2+}$ ions $\left(100 \mu \mathrm{M} \mathrm{ZnSO}_{4}\right)$ did not affect the degree of homo-dimerization of either wtHis-Cterminus or any of the other mutants (Figure 1E), suggesting that, unlike $\mathrm{K}^{+}$channels, the homotetramerization of RyR1 C-terminus is mediated by factors other than inter-coordination by $\mathrm{Zn}^{2+}$ ions at cysteine and histidine residues.

Because histidine can be positively charged at neutral $\mathrm{pH}, \mathrm{H} 5003$ may have participated in homodimerization of the $\mathrm{C}$-terminus through electrostatic interactions between or among C-terminal negatively charged residues. To test this hypothesis, negatively charged residues in the C-terminus ( 9 aspartic acids (D) and 12 glutamic acids (E) indicated by short bars in Figure $1 \mathrm{~A}$ ) were sub- stituted with alanines and the ability of the mutants to homo-dimerize was examined (Figure 2A). As before, the His-tag (Vector in Figure 2A) was expressed as a negative control. Ratios of the monomer- to dimer-band-intensities were normalized to that of wtHis-C-terminus (Wild) and were presented as bar graphs (Figure 2B). Similar to H5003A, the E4976A, D5026A, E5033A and D5034A mutants showed more than a $50 \%$ reduction in homo-dimerization ability compared with wtHis-C-terminus (E4976A by $56.2 \pm 9.9 \%$, $\mathrm{H} 5003 \mathrm{~A}$ by $94.2 \pm 1.6 \%, \mathrm{D} 5026 \mathrm{~A}$ by $54.7 \pm$ $7.4 \%$, E5033A by $95.6 \pm 1.4 \%$ and $\mathrm{D} 5034 \mathrm{~A}$ by $65.3 \pm 7.8 \%)$. Therefore it is likely that, along with $\mathrm{H} 5003$, these four negatively charged residues in the C-terminus of RyR1 play important roles in RyR1 homo-tetramerization via intra- and/or intermonomer electrostatic interactions. In support of this, Del-15, which lacks the last 15 amino acids of the C-terminus, including D5026, E5033 and $\mathrm{D} 5034$, also showed a $87.3 \pm 3.9 \%$ reduction in homo-dimerization ability compared with wtHis-Cterminus. This finding suggests that the loss of function previously reported in truncated RyR1s (deletion of the last 15 amino acids) (Gao et al., 1997) may have been caused by the inability of the truncated monomers to properly assemble and homo-tetramerize. According to the sequence alignment of RyR C-termini from different species

A

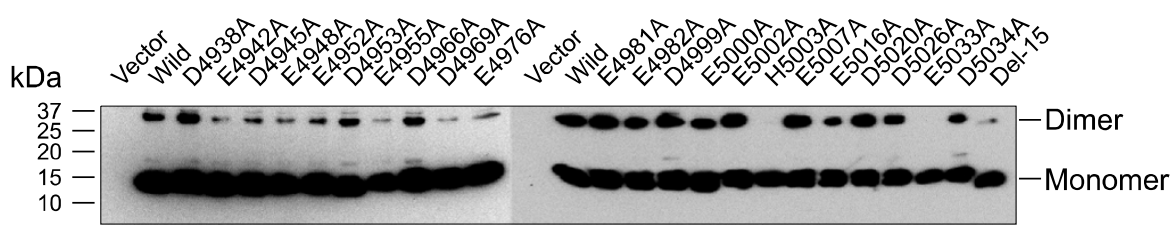

B

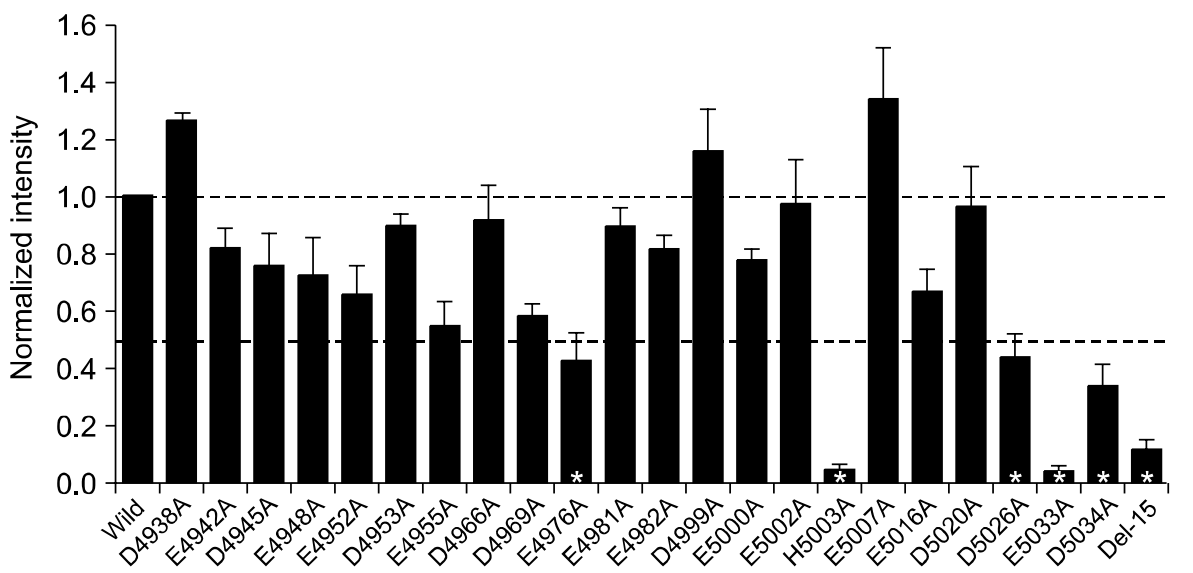

Figure 2. Homo-dimerization of a deletion mutant and single mutants of the negatively charged C-terminal residues. (A) Modified-native PAGE and immunoblot analysis of a deletion mutant (Del-15, deletion of the final 15 residues of the $\mathrm{C}$-terminus) and single mutants of the 21 negatively charged aspartic or glutamic acids. (B) Normalized ratios between monomer- and dimer-band-intensities by that of wtHis-C-terminus in (A) is presented as bar graphs. Mutants showing a reduction in homo-dimerization ability of $>50 \%$ are indicated by white asterisks. Wild is wtHis-C-terminus, as a positive control. Vector is His-tag only, as a negative control. 
(Supplemental data 3) and all three rabbit RyR isoforms (Figure $1 \mathrm{~A}$ ), negatively charged residues (D or E) at 5026, 5033 and 5034 are wellconserved in the RyR C-termini. In contrast, $I_{3} R$ $C$-termini vary in size and amino acid sequence. Therefore, despite similarities between the two sister $\mathrm{Ca}^{2+}$-release channels, the homo-dimerization and/or homo-tetramerization mechanisms of RyR1 are likely to be unique in at least some respects.

\section{The role of Helix I on the homo-dimerization of the C-terminus}

We used PSIpred from Brunel University to predict the secondary structure of the C-terminus. Using this program, two $\alpha$-helixes (Helix I and II) and a short $\beta$-strand were predicted for the $w t C$-terminus, with high confidence values (Figure 3A). According to the prediction, all five charged residues (E4976, D5003, D5026, E5033 and D5034) that caused a significant decrease in homo-dimerization ability when mutated to alanine (Figure 2) are located in random coils. This result suggests that the intraand/or inter-monomer electrostatic interactions at these residues have a degree of flexibility. The opening of RyR1s is associated with a 4-degree rotation of the TM assembly relative to the cytoplasmic assembly compared with the closed state (Orlova et al., 1996). As a result, the C-terminus just beyond the TM assembly of RyR1 may undergo re-location or conformational changes due to channel opening and closing. Therefore, because the charged residues are located in flexible random coils, they may either tolerate or facilitate the switching between channel opening and closing.

The effect of mutating all five charged residues was examined using the secondary structure prediction program (Supplemental data 2A) and the confidence-values changes caused by these mutations were checked. Interestingly, mutating all 5 residues induced confidence-value changes both in the 14 amino acids $(4,949-4,962)$ that comprised

A

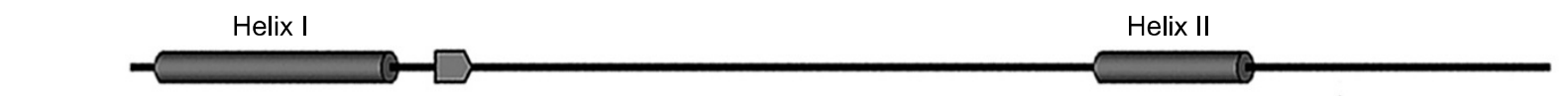

RYR1 4938 DAFGELRDQQEQVKEDMETKCFICGIGSDYFDTTPHGFETHTLEEHNLANYMFFLMYLINKDETEHTGQESYVWKMYQERCWDFFPAGDCFRKQYEDQLS 5037 $e^{x^{10}}$
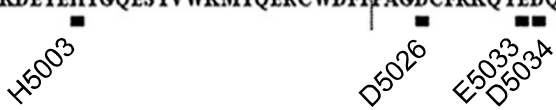

B

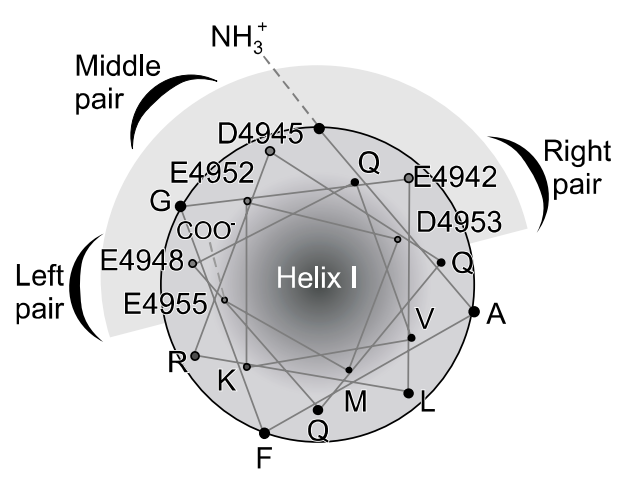

C

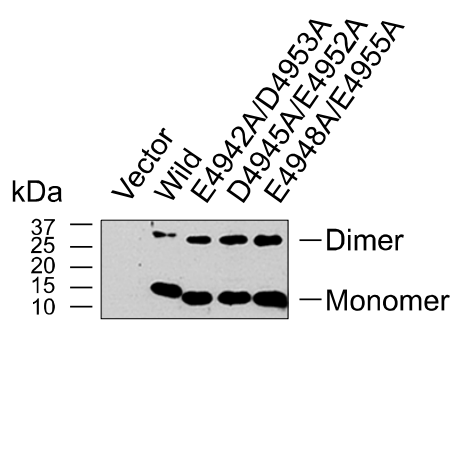

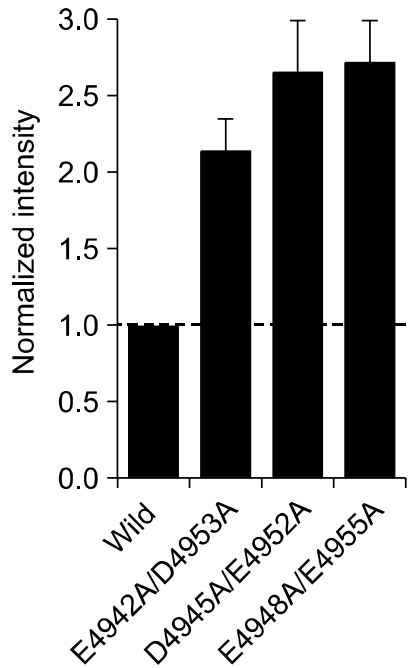

Figure 3. Prediction of the secondary structure of the C-terminus and double mutations at Helix I (A) According to the secondary structure prediction program described in Materials and methods, there were two predicted $\alpha$-helixes (Helix I and II, indicated by cylinders) and a short $\beta$-strand (indicated by a blunt arrow) in the C-terminus of RyR1. The dotted vertical line indicates the C-terminal end of Del-15. (B) N-terminal top view of Helix I. All six negatively charged residues of Helix I were located in one half of Helix I. (C) The homo-dimerization ability of double mutants at the negatively charged residues of the Helix I. Ratios between monomer- and dimer-band-intensities were normalized to that of the wtHis-C-terminus and were presented as bar graphs. Wild is wtHis-C-terminus, as a positive control. Vector is His-tag only, as a negative control. 
half of Helix I and in the mutated sites of the C-terminus, suggesting that, in addition to random coil interactions, Helix I also was involved in C-terminal homo-dimerization of RyRs.

The top view of right-handed Helix I containing six negatively charged residues is depicted in Figure 3B. Interestingly, all six residues (E4942, D4945, E4948, E4952, D4953 and E4955) face one half of Helix I and are paired in their directions (E4942/D4953 in the right, D4945/E4952 in the middle and E4948/E4955 in the left). When they mutated with a single alanine-substitution, their homo-dimerization ability was only marginally reduced (Figure 2). To determine if double alaninesubstitutions of the paired negatively charged residues had a greater effect, two negatively charged residues facing the same direction were substituted with alanines (Figure 3C). Unexpectedly, all three double-mutants showed an increase in homo-dimerization ability ( $>2$-fold), rather than decreased ability, compared with wtHis-C-terminus. According to secondary structure prediction (Supplemental data 2B), all three double-mutants showed a decrease in the confidence-value of
A

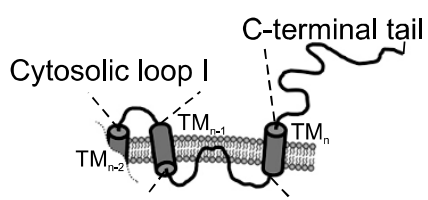

Putative pore-loop

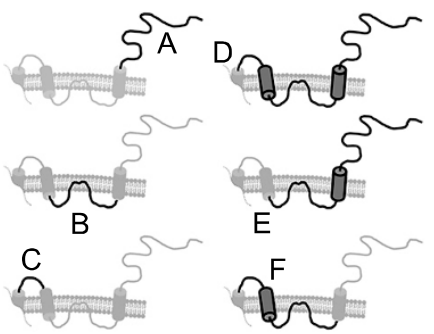

E

IP: RyR1 Ab

IB: RyR1 Ab

IB: His Ab

IP: RyR1 Ab

IB: RyR1 Ab

IB: GST Ab
B

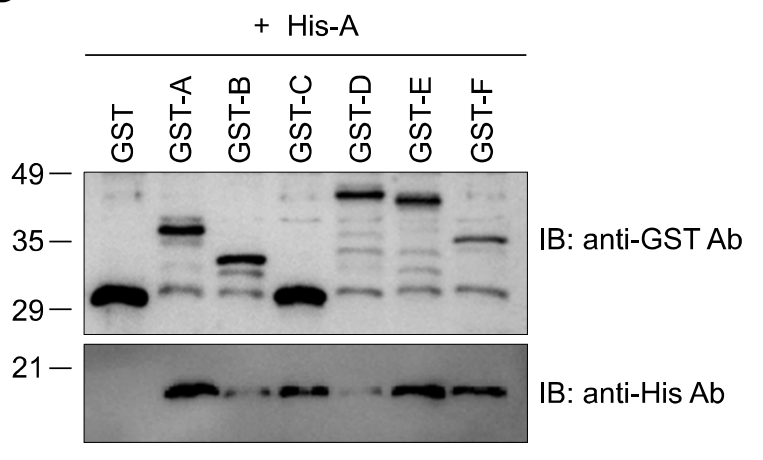

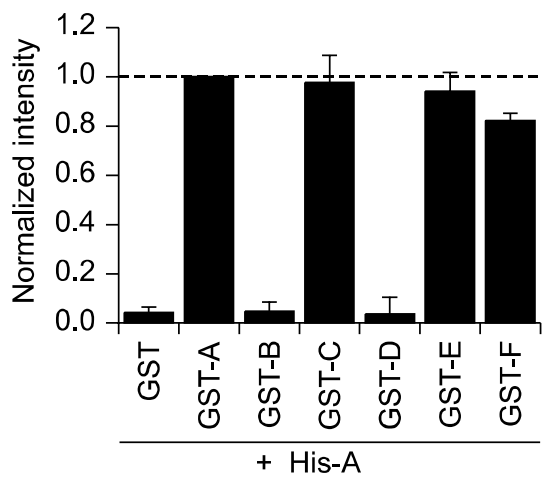

Figure 4. Interaction of the C-terminus with adjacent regions. (A) The pore-loop of RyR1 with the C-terminus was depicted based on a model proposed by Chen et al. (2002). The predicted last, second-last and third-last TM segments are represented as $\mathrm{TM}_{\mathrm{n}}, \mathrm{TM}_{\mathrm{n}-1}$ and $\mathrm{TM}_{\mathrm{n}-2}$, respectively. A, C-terminus; B, putative pore-loop; C, cytosolic loop I; D, cytoplasmic loop I to $\mathrm{C}$-terminus; $\mathrm{E}$, putative pore-loop to $\mathrm{C}$-terminus; F, cytoplasmic loop I to putative pore-loop. (B) Interaction between wtHis-A (wtHis-C-terminus, the same as Wild in Figures 1, 2 and 3 ) and each of the GST-tag proteins (GST-A, -B, -C, -D, -E and $-\mathrm{F}$ ) was examined using a GST-pull down assay. Each band intensity ratio was normalized to that of wtGST-A, wtGST-C or wthlis-A and was presented as bar graphs in the right side of (B), (C) and (D), respectively. (C) Interactions between wthis-A and single mutants at four positively charged amino acids in cytosolic loop I (GST-C mutants) were examined as described in (B). (D) GST-pull down assay of wtGST-C with each C-terminal mutant (His-A mutants) that showed a reduction in dimerization ability of $>50 \%$ (Figure 2). GST or His is GST-tag or His-tag only, as a negative control *Significant difference compared with wtGST-C or wtHis-A $(P<0.05)$. (E) Interaction between wtHis-A or wtGST-C and full-length RyR1 from solubilized rabbit skeletal SR was examined by co-immunoprecipitation with anti-RyR1 antibody and then by immunoblot analysis with anti-His or anti-GST antibody. Input (without RyR1 antibody) and GST (GST-tag only) were used as negative controls. 
Helix I, but an increase in the confidence-value of a random coil sequence $(4,984-N L A N Y M F F L M Y L I N-$ $4,997)$. This result indicates that the random coil sequence was stabilized by the double mutations in Helix I and, by so doing, enhanced the homodimerization ability of the C-terminus. This hypothesis agrees well with the fact that Del-15, which had a reduction in homo-dimerization ability > $87 \%$, had a decrease in confidence-value of the same random coil region (Supplemental data $2 \mathrm{~A}$ ). Therefore, in addition to the five charged residues in Figure 2, Helix I also must play an important role in the homo-tetramerization of RyR1 via homodimerization of the $\mathrm{C}$-terminus.

\section{Interaction between the C-terminus and cytosolic loop I}

The C-terminus is well equipped to interact with neighboring regions of RyR1 due to its location at the free end of the RyR1 and its predicted domi- nant flexible random coils. To test this possibility, proteins longer or shorter than the C-terminus were cloned as N-terminal GST-tag proteins (Figure 4A): GST-A, C-terminus (D4,938-S5,037, the same as His-C-terminus except for GST-tag); GST-B, putative pore-loop (R4,860-D4,917, a SR luminal portion between $\mathrm{TM}_{\mathrm{n}-1}$ and $\mathrm{TM}_{\mathrm{n}}$ ); GST-C, cytosolic loop I (K4,821-K4,835, a short cytoplasmic loop between $\mathrm{TM}_{\mathrm{n}-2}$ and $\left.\mathrm{TM}_{\mathrm{n}-1}\right)$; GST-D, cytoplasmic loop I to C-terminus (K4,821-S5,037); GST-E, putative pore-loop to C-terminus (R4,860-S5,037); GST-F, cytoplasmic loop I to putative pore-loop (K4,821-D4,917).

Interaction between wtHis-A (wtHis-C-terminus, the same as Wild in Figures 1,2 and 3) and one of the individual GST-tag proteins was examined using the GST pull-down assay (Figure 4B). GSTA, GST-C, GST-E and GST-F showed strong interactions with wtHis-A. Interaction of His or the GST tag itself with the control proteins was not detected (His and GST in Figure 4B, C, and D). As
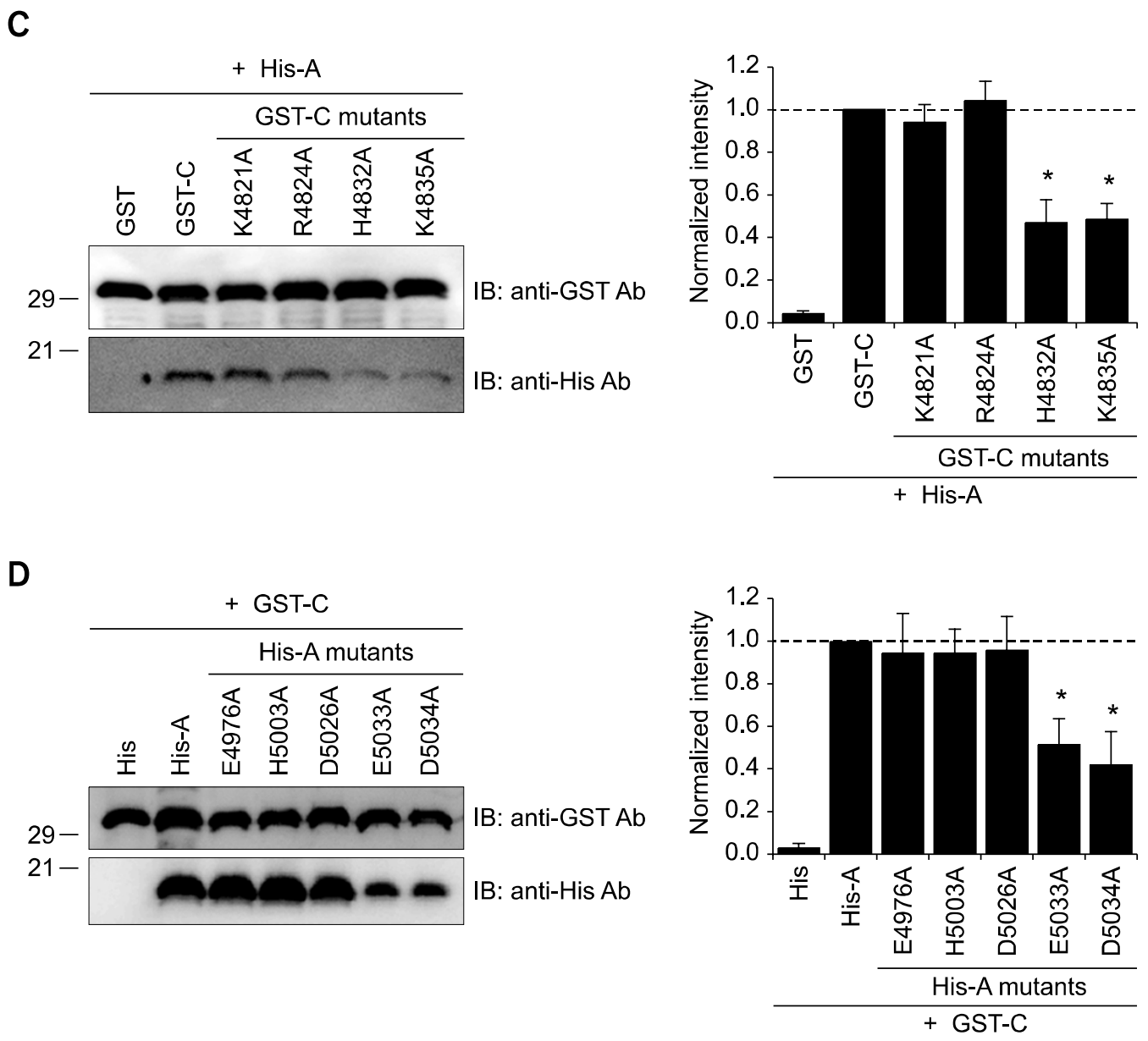

Figure 4. Continued. 
expected from previous results, there were robust interactions between His-A and GST-A or between His-A and GST-E, and that most likely was due to interactions between the 'A' portions (C-termini) of the proteins. Interestingly, a robust interaction between His-A and GST-C or between His-A and GST-F also was observed. Based on the makeup of these two constructs, this interaction may best be explained by an interaction between the ' $A$ ' portion of His-A and the ' $C$ ' portion (cytosolic loop I) of GST-C and GST-F because the GST-C and GST-F share this structural feature. Cytoplasmic loop I is part of Region $3(3,916-4,973)$, one of the mutational hot-spots associated with malignant hyperthermia and central core disease (Lyfenko et al., 2004). Mutations at Region 3 are pathological because they presumably alter the assembly of the pore-forming region and consequently change the selectivity or gating properties of RyR1. The shift of GST-D, which contained both 'A' and ' $C$ ' portions, was likely caused by self-folding within a single GST-D molecule. This self-folding would prevent interaction between His-A and GST-D, as was observed in the current experiment. In terms of full-length RyRs, either self (intra)-folding between ' $A$ ' and ' $C$ ' portions in a single RyR1 or inter-folding between ' $A$ ' and ' $C$ ' portions from two RyR1s could be possible. There was no significant interaction between His-A and GST-B.

Cytosolic loop I contains four charged residues and all of them are positively charged. To identify residues in cytoplasmic loop I that are critical for interaction with the C-terminus (wtHis-A), each of the four positively charged residues was substituted with alanine and then subjected to the GST pull-down assay with wtHis-A (Figure 4C). Two of the mutants, H4832A and $\mathrm{K} 4835 \mathrm{~A}$, showed a significantly decreased ability to interact with wtHis-A. Conversely, wt cytoplasmic loop I (wtGSTC) was subjected to GST-pull down assays with each of the five His-A mutants that showed more than a $50 \%$ reduction in homo-dimerization ability, as shown in Figure 2 (Figure 4D). E5033A and D5034A had a significant reduction in their ability to interact with wtGST-C. Therefore, it appears that positively charged $\mathrm{H} 4832$ and K4835 could be electrostatic partners with negatively charged E5033 and D5034, forming salt bridges, in the interaction between cytoplamsic loop I and the C-terminus.

According to the secondary structure prediction for cytoplasmic loop I (Supplemental data 4), E5033 and D5034 also were located in a random coil, as were the five critical charged residues shown in Figure 3A. Because the proposed mechanism involves all seven charged residues in 'flexible random coils' and because of the effect of mutation in Helix I on the 'flexible random coil', the homo-tetramerization of RyR1 via its C-terminal electrostatic interactions among RyR1 monomers is probably governed by an 'induced-fit model'. In this model, C-terminus has complete structural arrangements when they are gathered and stand in a knot (a tetrameric RyR1). In this context, if the mechanism for RyR1 homo-tetramerization is accomplished by dimerization of two dimers, creation of the tetramer requires more than homodimerization of two monomers, probably due to volume effects and steric hindrances. Therefore, the involvement of other RyR1 regions, such as $\mathrm{N}$-terminal foot regions, cannot be completely excluded as playing a role in the homo-tetramerization of RyR1.

Because the homo-tetramerization mechanism of RyR1 was examined using only its C-terminus and cytoplasmic loop I fragments, it is possible that the structures of these fragments are different than the corresponding regions of full-length RyR1 and may negate the findings of the present study. To rule out this possibility, co-immunoprecipitation experiments of full-length RyR1 were performed with both fragments - confirming the interaction of both the C-terminus (wtHis-A) and cytoplasmic loop I (wtGST-C) with full-length RyR1 (Figure 4E), thus supporting the original hypothesis and more possibility for inter-folding by ' $A$ ' and ' $C$ ' portions from two full-length RyR1s instead of self (intra)folding by ' $A$ ' and ' $C$ ' portions in a single full-length RyR1.

\section{Acknowledgments}

This work was supported by the Korea Research Foundation Grant funded by the Korean Government (MOEHRD, Basic Research Promotion Fund, KRF-2006-331-E00015) and the Catholic Medical Center Research Foundation Grant (2007) to E.H.L., and NIH AR43140 and AR17605 to P.D.A. We acknowledge Drs. Isaac N. Pessah and Jose R. Lopez for their kind review and editorial suggestions.

\section{References}

Bixby KA, Nanao MH, Shen NV, Kreusch A, Bellamy H, Pfaffinger PJ, Choe S. $\mathrm{Zn}^{2+}$-binding and molecular determinants of tetramerization in voltage-gated $\mathrm{K}^{+}$channels. Nat Struct Biol 1999;6:38-43

Chen SR, Li P, Zhao M, Li X, Zhang L. Role of the proposed pore-forming segment of the $\mathrm{Ca}^{2+}$ release channel (ryanodine receptor) in ryanodine interaction. Biophys $\mathrm{J}$ 2002;82:2436-47

Galvan DL, Borrego-Diaz E, Perez PJ, Mignery GA. Subunit 
oligomerization, and topology of the inositol 1,4, 5-trisphosphate receptor. J Biol Chem 1999;274:29483-92

Gao L, Tripathy A, Lu X, Meissner G. Evidence for a role of C-terminal amino acid residues in skeletal muscle $\mathrm{Ca}^{2+}$ release channel (ryanodine receptor) function. FEBS Lett 1997;412:223-6

Hakamata Y, Nakai J, Takeshima H, Imoto K. Primary structure and distribution of a novel ryanodine receptor/calcium release channel from rabbit brain. FEBS Lett 1992;312: 229-35

Jahng AW, Strang C, Kaiser D, Pollard T, Pfaffinger P, Choe $\mathrm{S}$. Zinc mediates assembly of the T1 domain of the voltage-gated K channel 4.2. J Biol Chem 2002;277:4788590

Laemmli UK. Cleavage of structural proteins during the assembly of the head of bacteriophage T4. Nature 1970; 227:680-5

Lee EH, Rho SH, Kwon SJ, Eom SH, Allen PD, Kim DH. $\mathrm{N}$-terminal region of FKBP12 is essential for binding to the skeletal ryanodine receptor. J Biol Chem 2004;279:26481-8

Lyfenko AD, Goonasekera, SA, Dirksen RT. Dynamic alterations in myoplasmic $\mathrm{Ca}^{2+}$ in malignant hyperthermia and central core disease. Biochem Biophys Res Commun 2004;322:1256-66

Magnino F, Schmidt K, Mery L, Dufour JF. Rat inositol 1,4,5-trisphosphate receptor isoform 2 interacts with itself in its C-terminal portion and upstream of the first transmembrane domain. Eur J Biochem 2001;268:5981-8

McGuffin LJ, Bryson K, Jones DT. The PSIPRED protein structure prediction server. Bioinformatics 2000;16:404-5

Ogawa Y, Kurebayashi N, Murayama T. Putative roles of type 3 ryanodine receptor isoforms (RyR3). Trends Cardiovasc Med 2000;10:65-70

Orlova EV, Serysheva II, van Heel M, Hamilton SL, Chiu W. Two structural configurations of the skeletal muscle calcium release channel. Nat Struct Biol 1996;3:547-52

Rushmore TH, Pickett CB. Glutathione S-transferases, structure, regulation, and therapeutic implications. J Biol Chem 1993; 268: 11475-8

Samso M, Wagenknecht T, Allen PD. Internal structure and visualization of transmembrane domains of the RyR1 calcium release channel by cryo-EM. Nat Struct Mol Biol 2005;12:539-44

Sandow A. Excitation-contraction coupling in skeletal muscle. Pharmacol Rev 1965; 17:265-320

Stewart R, Zissimopoulos S, Lai FA. Oligomerization of the cardiac ryanodine receptor C-terminal tail. Biochem J 2003;376:795-9

Williams AJ, West DJ, Sitsapesan R. Light at the end of the $\mathrm{Ca}^{2+}$-release channel tunnel: structures and mechanisms involved in ion translocation in ryanodine receptor channels. Q Rev Biophys 2001;34:61-104

Zorzato F, Fujii J, Otsu K, Phillips M, Green NM, Lai FA, Meissner G, MacLennan DH. Molecular cloning of cDNA encoding human and rabbit forms of the $\mathrm{Ca}^{2+}$ release channel (ryanodine receptor) of skeletal muscle sarcoplasmic reticulum. J Biol Chem 1990;265:2244-56 